\title{
Predictions of Indoor Thermal Comfort Level using Fuzzy Logic
}

\author{
Sandeep Kumar Manocha ${ }^{1}$, Hari Kumar Singh ${ }^{2}$, P.K. Yadav ${ }^{3}$, B.M. Suman ${ }^{4}$ \\ Suresh Gyan Vihar University, Mahal Jagatpura, Jaipur, Rajasthan, \\ Central Building Research Institute, - Roorkee.
}

\begin{abstract}
Prediction of Thermal Comfort Level (TCL) is one of the intricate observable facts to understand. This is due the fact that thermal comfort level is quite complicated to demonstrate precisely in view of the fact that the factual procedure has involvement of the human beings. For analytical principles, it can be believed that the individual is a "black box" which obtains inputs as atmospheric provisions and personage variables, while the output is the subjective acuity of thermal comfort level. Interior comfort state of a building relies on the peripheral ambiance and thermo-physical properties of the materials employed in the building. The outer climatologically constraints can be collective as a sole constraint known as Sol Air Temperature (SAT). Similarly, internal and open air surface heat transfer coefficients and thermal properties of the materials employed in the buildings are united in a single feature, termed as Overall Thermal Transmittance (OTT). In the present study, a fuzzy logic move towards achieving the suitable thermal comfort level is being employed assuming the SAT and OTT as input constraints. The complication of the human intellectual and the imprecision of linguistic expressions are taken into the deliberation. A comprehensive clarification of the relevance uniting linguistic approach to the optimization under numerous thermal provision principles is presented in this study.
\end{abstract}

Keywords: Sol Air Temperature, Thermal Comfort Level, Overall Thermal Transmittance, Fuzzy Logic.

\section{Introduction}

Energy effectiveness in the buildings is an attractive matter at present because it contributes concurrently to the diminution of conventional fuels utilization; energy reduces cost for the building owners and lessens in the global warming gas liberated in the surroundings. In whichever case, energy efficiency must never compromise indoor thermal comfort level (TCL) for the building occupants [4]. TCL is defined as the state of individual mind that articulates contentment with the surrounding environment (ANSI/ASHARE standard 55) [1]. The imperative objective of HVAC (heating, ventilation, and air conditioning) for design engineers is to preserve the customary of thermal comfort for the occupants in the buildings or other inclusions.

There are numerous aspects which influence the TCL through heat conduction, convection, radiation, and evaporative heat loss. These constraints provide some information about the optimal circumstances and what can be done in the vicinity to advance comfort levels in moderately warm or cool interior region. Thermal comfort is preserved when the heat produced by individual metabolism is permitted to dispel, thus persuading thermal equilibrium with the surroundings. It has been identified that the commotion of reacting hot or cold is not just reliant on air temperature alone.

Commonly individual apprehensions about interior thermal comfort arise in the regions that are inadequately ventilated and /or poorly sheltered from the sunlight. Personage thermal comfort can also be influenced by bodily action, packed working regions and some remedial situations.

Thermal comfort is a prejudiced judgment, and even in most favorable circumstances some people might experience discomfort. Therefore, preserving an environment to the level of thermal comfort level (TCL) is significant for the healthiness and comfort of human.

TCL is quite complicated to present mathematically since the actual practice has the participation of individual attentiveness. It can be assumed that the human is a "black box" that receives environmental conditions and personal variables as input, whereas output is the subjective perception of TCL which is shown in the figure given below.

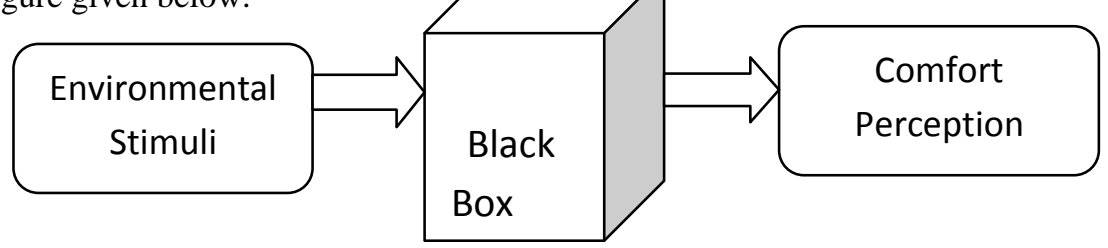

\section{Fig.1 Black box process of human perception}

Comfort is a fuzzy perception, diverse for different people. A design of fuzzy system for living space thermalcomfort regulation has been explained by Dounis A.I. et al [3]. Design and estimation for building occupants thermal-visual comfort and indoor air quality contentment have been deliberated by Kolokotsa D et al [4]. The 
advanced fuzzy logic controllers like fuzzy PID, fuzzy PD and adaptive fuzzy PD control is employed while designing the thermal-visual comfort and interior air quality contentment. Recently Singh et al. [5] has designed a fuzzy controller for cabin to control the cabin temperature and introduced a concept of fuzzy controller system to control thermal comfort of vehicle passenger.

A latest logical move towards the process control is explained with particular focus on human factor, by employing the measurement metric, which is a constituent part of the feedback loop by Zoran L. Baus et al [6]. For the predictable thermal comfort preservation practice control, a correction is provided in accordance with personage experience, while preserving indication values within boundaries acceptable by the applicable standard. The fuzzy sequence control is deliberated incorporating the personage commotion of comfort. A comfort observing system and hazard revealing unit using fuzzy logic is explained by the Tennakoon et al [7]. The initiative after the comfort observing system is to support the user to get a proposal how various aspects such as room temperature, humidity, airflow and clothing influence the human comfort. In this paper, a fuzzy logic move is employed to predict an accurate TCL assuming the SAT and OTT as input constraints. A comprehensive exposition of the relevance uniting linguistic approach to the optimization under multiple thermal circumstances criterion is presented in this study.

\section{Fuzzy Logic Modeling}

There are number of different methods are available some of the method require a comparatively truthful model in order to develop a satisfactory system. The concept of fuzzy set theory is a membership function, which represents numerically to what degree an element belongs to a set. In fuzzy set theory, an element can be a member of a particular set to a certain degree and at the same time be a member of a different set to a certain degree. To what degree an element belongs to a certain set is called the membership degree. In fuzzy rule-based systems, knowledge is represented by if-then rules. Fuzzy rules consist of two parts: an antecedent part stating conditions on the input variable(s) and a consequent part describing the corresponding values of the output variable(s) [11]. The aim of this study was to construct fuzzy knowledge-based models for the predictions of indoor thermal comfort level (based on the Mamdani) approach. Fuzzy modeling involves the following three steps:

i. Fuzzification

ii. Fuzzy Rule Inference

iii. Defuzzification

\subsection{FUZZIFICATION}

The fuzzification comprises the process of transforming crisp values into grades of membership for linguistic terms of fuzzy sets. The Membership Function (MF) is used to associate a grade to each linguistic term. The terminology of linguistic variable was introduced by Zadeh [12] as an approach to capture natural experience commonly used by controller black box.

\subsection{FUZZY RULE INFERENCE}

Fuzzy rule inference method is used to define some sets of fuzzy logic operators along with production rules. The most common rule is called IF-THEN Rule, which can be used to formulate the conditional statements that comprise fuzzy logic.

\subsection{DEFUZZIFICATION}

In Defuzzification, Fuzzy sets act as the input in the process and output is the single number. The aggregate of fuzzy set encompasses a range of output values and so must be defuzzified in order to get the single output value from the set. A Conceptual Fuzzy Logic Modeling Approach has been depicted in Figure 2.

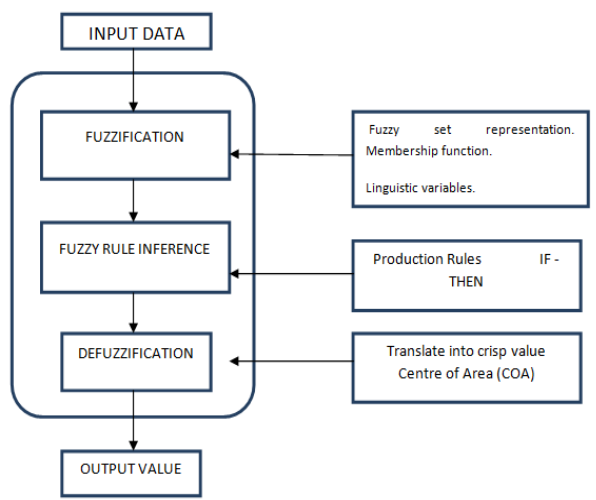

Fig2. Intangible Fuzzy Logic Modeling 


\section{Fuzzy Inference Engine (Fie)}

Fuzzy inference is the process of formulating the mapping from a given input set to an output using fuzzy logic. Fuzzy logic [11]-[13] is a superset of conventional Boolean logic and extends it to deal with new aspects such as partial truth and uncertainty. The basic elements of fuzzy logic are linguistic variables, fuzzy sets, and fuzzy rules [14]. The linguistic variables' values are words, specifically adjectives like "very low", "low", "medium", "high", and "very high" A fuzzy set is a collection of couples of elements. It generalizes the concept of a classical set, allowing its elements to have a partial membership. The degree to which the generic element " $\mathrm{x}$ " belongs to the fuzzy set A (expressed by the linguistic statement $\mathrm{x}$ is A) is characterized by a membership function $(\mathrm{MF}), \mathrm{f}_{\mathrm{A}}(\mathrm{x})$. The membership function of a fuzzy set corresponds to the indicator function of the classical sets. It can be expressed in the form of a curve that defines how each point in the input space is mapped to a membership value or a degree of truth between 0 and 1 . The most common shape of a membership function is triangular, although trapezoidal and bell curves are also used. This operation normalizes all inputs to the same range and has a direct effect on system performance and accuracy. A fuzzy set "A" defined within a finite interval called universe of discourse $\mathrm{Z}$ as follows: $\left.\mathrm{A}=\left\{\left(\mathrm{x}, \mathrm{f}_{\mathrm{A}}(\mathrm{x})\right), \mathrm{f}(\mathrm{x})\right): \mathrm{Z} \rightarrow[0,1]\right\}$

$\mathrm{Z}$ is the whole input range allowed for a given fuzzy linguistic variable. All fuzzy sets related to a given variable make up the term set, the set of labels within the linguistic variable described or, more properly, granulated. Fuzzy rules form the basis of fuzzy reasoning. They describe relationships among imprecise, qualitative, linguistic expressions of the system's input and output. Generally, these rules are natural language representations of human or expert knowledge and provide an easily understood knowledge representation scheme.

A perception of estimate interpretation was introduced by Zadeh in 1975. This perception provides a strong outline for interpretation in the visage of inaccurate and tentative information. The accomplishment of the fuzzy IF- THEN rule is the origin of this theory, which is a mathematical analysis of the linguistic IF-THEN rule. A linguistic IF-THEN rule is a linguistic verdict that is written in effortless form such as:

If " $\mathrm{X}$ " is $\mathrm{A}$ and " $\mathrm{Y}$ " is $\mathrm{B}$ then " $\mathrm{Z}$ " is $\mathrm{C}$

Here $\mathrm{X}, \mathrm{Y}, \mathrm{Z}$, are variables and $\mathrm{A}, \mathrm{B}, \mathrm{C}$ are the corresponding linguistic values. The rules identify the names of the variables $\mathrm{X}$, are variables and $\mathrm{A}, \mathrm{B}, \mathrm{C}$ are the corresponding linguistic values. The rules identify the names of the variables $\mathrm{X}, \mathrm{Y}, \mathrm{Z}$, with the universe in which the fuzzy values $\mathrm{A}, \mathrm{B}$, and $\mathrm{C}$ live.

There are two types of fuzzy inference models:

1. Mamdani [11],

2. TSK or Sugeno [12].

Interpreting an if-then rule involves two distinct parts: first evaluating the antecedent and then applying results to the consequent (known as implication). In the case of two-valued or binary logic, if-then rules do not present much difficulty. If the premise is true, then the conclusion is true, whereas with fuzzy approach, if the antecedent is true to some degree of membership, then the consequent is also true to that same degree.

Mamdani-type [12] inference expects the output membership functions to be fuzzy sets. After the aggregation process, there is a fuzzy set for each output variable that needs defuzzification.

It is possible, and in many cases much more efficient, to use a single spike as the output's membership function rather than a distributed fuzzy set. This is sometimes known as a singleton output membership function, and it can be thought of as a pre-defuzzified fuzzy set. It enhances the efficiency of the defuzzification process because it greatly simplifies the computation required by the more general Mamdani method, which finds the centroid of a two-dimensional function. Rather than integrating across the two-dimensional function to find the centroid, Sugeno-type systems use weighted sum of a few data points. In general, Sugeno-type systems can be used to model any inference system in which the output membership functions are either linear or constant.

\section{Proposed Model}

As shown in the fig. 3, the two key aspects SAT and OTT are believed for the prediction of TCL. The input variables SAT and OTT which are supposed to have insightful effect on TCL prediction are employed to assist the optimum temperature difference which then will be considered as level of thermal comfort.

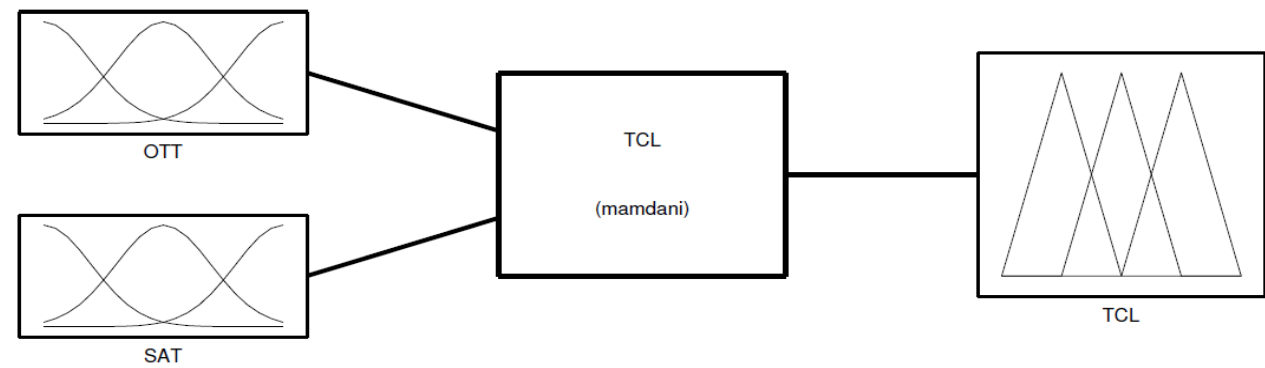

Figure3. Inference system block diagram 
In the present study membership function for SAT and OTT are taken to represent the level of thermal comfort and the output of the inference engine may be employed to direct the thermal temperature inside a building.

\subsection{SOL-AIR TEMPERATURE (SAT)}

SAT is defined as the temperature under provisions of no unswerving solar radiation and no air action, which would cause the same amount of heat transfer into a house or a building as that caused by the interaction of all existing environmental circumstances.

$\mathrm{SAT}=\mathrm{T}_{\mathrm{OA}}+\alpha \mathrm{I} / \mathrm{h}_{\mathrm{o}}-\mathrm{Eil} / \mathrm{h}_{\mathrm{o}}$

Here, $\mathrm{T}_{\mathrm{OA}}=$ Outdoor air temp,

$\alpha=$ absorptivity of external surface,

I= Direct solar radiations,

$\mathrm{E}=$ Emissivity,

il $=$ diffused solar radiations,

$\mathrm{h}_{\mathrm{o}}=$ Outside surface heat transfer coefficient,

Eil is $85 \mathrm{~W}$ for roof and for wall it is zero.

\subsection{OVERALL THERMAL TRANSMITTANCE (OTT)}

OTT (also known as the $U$ factor) is the rate of heat flow per unit square area of unit thickness, when temperature difference of $1^{\circ} \mathrm{C}$ is preserved between the surroundings separated by the slab. It involves thermal properties of the materials employed in the building, interior and open-air surface heat transfer. In arithmetical terms, the U- factor is the reciprocal value of the total resistance. The $\mathrm{U}$ - factor is only a suggestion of the conduction rate of heat transfer, it is a quantitative dimension of how fine heat conduction through a roof or wall for a given temperature difference.

OTT $=\left(\mathrm{h}_{\mathrm{i}}^{-1}+\left(\sum(\mathrm{L} / \mathrm{K})_{\mathrm{I}}\right)+\mathrm{h}_{\mathrm{o}}^{-1}\right)^{-1}$

Here, $L_{i}=$ Thickness of inside surface,

$\mathrm{K}_{\mathrm{i}}=$ Thermal conductivity, and

$\mathrm{h}_{\mathrm{i}}=$ Inside surface heat transfer coefficient.

* Levels of SAT and OTT in terms of temperature and U-value respectively

\begin{tabular}{|c|c|c|}
\hline Level & $\mathrm{SAT}\left({ }^{0} \mathrm{C}\right)$ & $\mathrm{OTT}\left(\mathrm{W} / \mathrm{m}^{2} \mathrm{~K}\right)$ \\
\hline Very low & $\leq 20$ & $\leq 0.5$ \\
\hline Low & $20-30$ & $0.5-1.75$ \\
\hline Medium & $30-40$ & $1.75-3.0$ \\
\hline High & $40-50$ & $3.0-4.25$ \\
\hline Very high & $\geq 50$ & $\geq 4.25$ \\
\hline
\end{tabular}

\subsection{MEMBERSHIP FUNCTIONS}

For the $f_{A}(x)$ as the Membership Function (MF), a huge category of functions can be taken like triangular, trapezoidal, Gaussian and bell functions. Nevertheless triangular membership function has been selected for its simplicity of utilize in fuzzy dedicated hardware. MFs measured for SAT and OTT are "Very Low", "Low", "Medium", "High" and "Very High" represented by 5 triangular curves for this model shown in fig. 4 and 5 and TCL is taken as 5 curves of Gaussian MF "Hot", "Warm", "Slightly Warm", "Slightly Cold", and "Cold" shown in fig. 6.

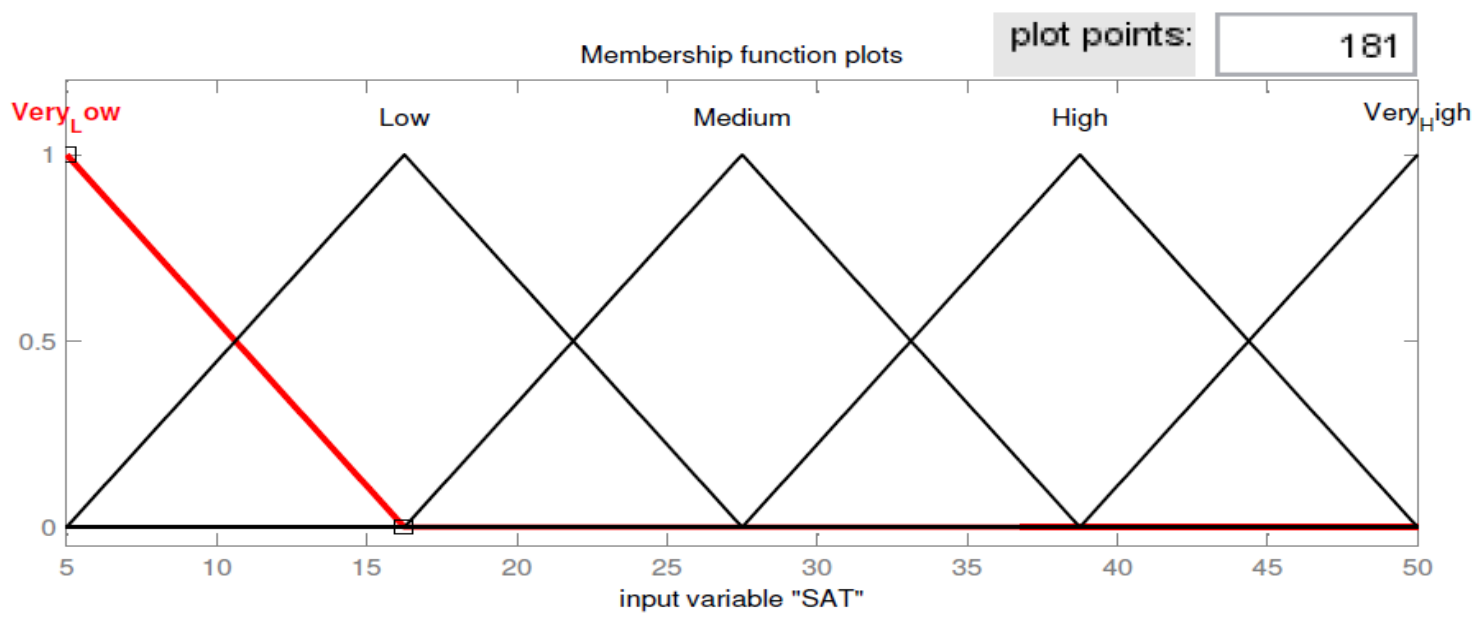

Fig.4 Fuzzy position equivalent to SAT 


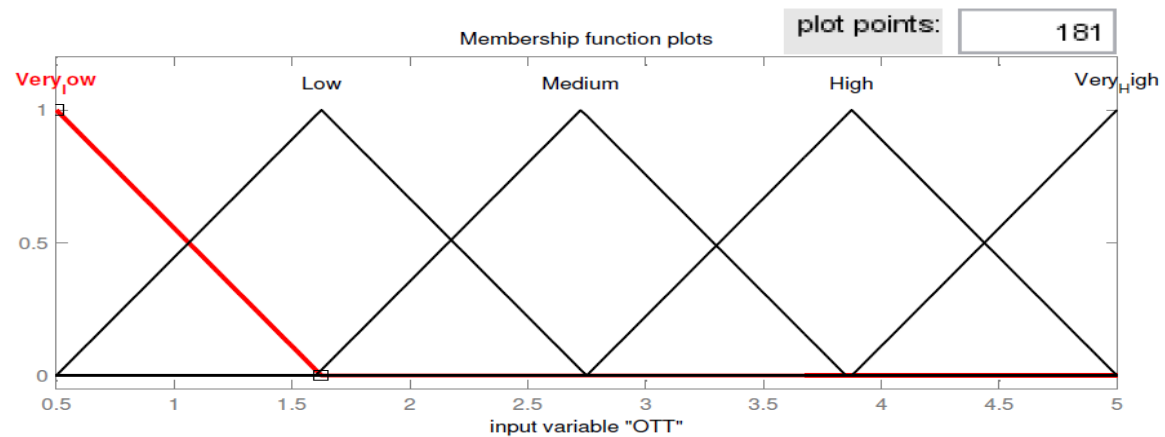

Fig.5 Fuzzy position equivalent to OTT

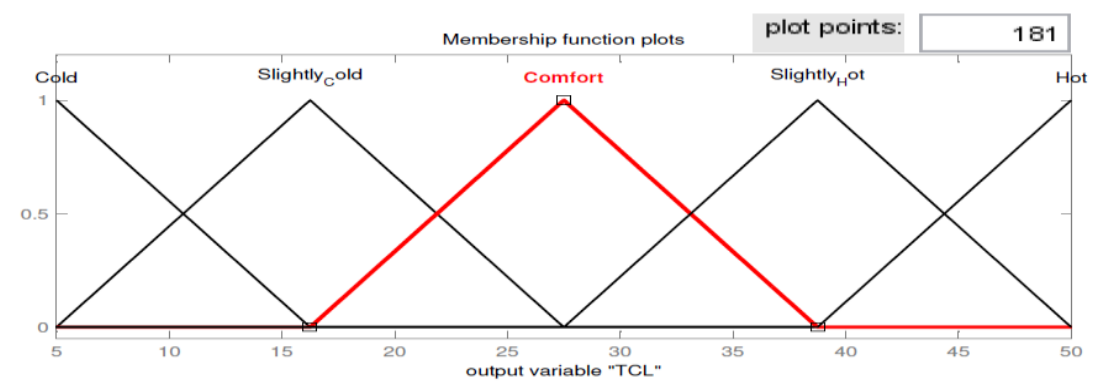

Fig.6 Fuzzy position equivalent to TCL

When multiple input combinations are to get executed, the basic concept of switching and time slices comes into picture. In our proposed algorithm as shown under, a newly sat (), and ott ()

value, will be added to the input queue. This queue consists of the remaining input values from last cycle that has not yet been executed

Loop

For each state of temperature, do the following:

1. For each value of comfort condition feed its sol air temperature (sat) and overall thermal transmittance (ott) into the inference engine. Consider the output of inference module as thermal comfort level.

2. Store the values of thermal comfort level in an array tcl().

3. Execute the corresponding tcl () value of highest magnitude until a scheduling event occurs.

4. Update the system states.

End Loop

\section{Results And Discussions}

Fuzzification of the used parameter is made by aid follows functions. These formulas were determined by using assumed values.

$\mu_{V H}\left(\mathrm{i}_{1}\right)=\left\{\begin{array}{l}0 ; \mathrm{i}_{1}<38.75 \\ \frac{50-i_{1}}{11.25} ; 38.75 \leq \mathrm{i}_{1} \leq 50 \\ 1 ; \mathrm{i}_{1}>50\end{array}\right\}$

$\mu_{V L}\left(\mathrm{i}_{1}\right)=\{1 / 5+0.5 / 10.625+0 / 16.25\}$

For example, for SAT $=27.5$ and OTT $=2.188$, the rules 12 and 13 will be applied which notices that $\mu_{M}(\mathrm{SAT})$ $=1$ but that the other membership functions for the SAT inputs are all "off" (i.e., their values are zero). For the OTT input it is seen that $\mu_{L}$ (OTT) $=0.5$ and that the other membership functions are off.

\section{FOR RULE 12}

The premise (slightly cold), we have $\mu_{\text {slightly cold }}(12)=\min \left\{\mu_{m}(\mathrm{SAT}), \mu_{L}(\mathrm{OTT})\right\}=\min \{1,0.5\}=0.5$, by using the minimum of the two membership values. The notation $\mu_{12}$ represents for rule $\{12\}$ so that we are 0.5 certain that this rule applies to the current situation. This rule indicates that if its premise is true then the 
action indicated by its consequent should be taken. For rule(12) the main consequent is "TCL is slightly cold". The membership function for the conclusion reached by rule (12), which is denoted as $\mu_{12}$ (TCL) $=\min \left\{0.5, \mu_{M}\right.$ (TCL) $\}$. This membership function defines the implied fuzzy set for rule $\{12\}$.

\section{FOR RULE 13}

The premise (comfort), we have $\mu_{\text {comfort }}(13)=\min \left\{\mu_{M}\right.$ (SAT), $\left.\mu_{M}(\mathrm{OTT})\right\}=\min \{1,0.5\}=0.5$, by using the minimum of the two membership values. So that we are 0.5 certain that this rule applies to the current situation. This rule indicates that if its premise is true then the action indicated by its consequent should be taken. For rule (13) the consequent is "TCL is comfort". The membership function for the conclusion reached by rule (13), which is denoted as $\mu_{13}$, is given by $\mu_{13}$ (TCL) $=\min \left\{0.5, \mu_{H}\right.$ (TCL $\}$.

This membership function defines the implied fuzzy set for rule $\{13\}$. Is is noticed that we are certain that both rule (12) and (13) apply to the current situation. From mamdani max-min inference, the membership function of system will be found as $\max \left(\mu_{12}\right.$ and $\left.\mu_{13}\right)=0.5$ and then defuzzification operation is applied to the final component of the fuzzy controller produced by the inference mechanism and combines their effects to provide the "most certain" controller output. Then the output denoted by "TCL" can be calculated that best represents the conclusions of the fuzzy controller that are represented with the implied fuzzy sets.

\section{CENTRE OF GRAVITY FOR SINGLETONES:}

The output membership values are multiplied by their corresponding singleton values and then are divided by the sum of membership values.

\section{$T C L^{\text {crisp }}=\sum \mathrm{xi} \mu_{i}(\mathrm{x}) / \sum \mu_{i}(\mathrm{x})$ \\ MEMBERSHIP FUNCTIONS:}

$$
\begin{aligned}
& \mu_{V L}\left(\mathrm{i}_{1}\right)=\left\{\begin{array}{l}
0 ; \mathrm{i}_{1}<5 \\
\frac{16.25-i_{1}}{11.25} ; 5 \leq \mathrm{i}_{1} \leq 16.25 \\
0 ; \mathrm{i}_{1}>16.25
\end{array}\right\} \\
& \mu_{V L}\left(\mathrm{i}_{1}\right)=\{1 / 5+0.5 / 10.625+0 / 16.25\} \\
& \mu_{L}\left(i_{1}\right)=\left\{\begin{array}{l}
\frac{i_{1}-5}{11.25} ; 5 \leq i_{1} \leq 16.25 \\
\frac{27.5-i_{1}}{11.25} ; 16.25 \leq i_{1} \leq 27.5 \\
0 ; i_{1}>27.5
\end{array}\right\} \\
& \mu_{L}\left(i_{1}\right)=\{0 / 5+0.5 / 10.625+1 / 16.25+0.5 / 21.875+0 / 27.5\} \\
& \mu_{M}\left(i_{1}\right)=\left\{\begin{array}{l}
\frac{i_{1}-16.25}{11.25} ; 16.25 \leq i_{1} \leq 27.5 \\
\frac{38.75-i_{1}}{11.25} ; 27.5 \leq i_{1} \leq 38.75 \\
0 ; i_{1}>38.75
\end{array}\right\} \\
& \mu_{M}\left(\mathrm{i}_{1}\right)=\{0 / 16.25+0.5 / 21.875+1 / 27.5+0.5 / 33.125+0 / 38.75\} \\
& \mu_{H}\left(\mathrm{i}_{1}\right)=\left\{\begin{array}{l}
\frac{\mathrm{i}_{1}-27.5}{11.25} ; 27.5 \leq \mathrm{i}_{1} \leq 38.75 \\
\frac{50-i_{1}}{11.25} ; 38.75 \leq \mathrm{i}_{1} \leq 50 \\
0 ; \mathrm{i}_{1}>50
\end{array}\right\} \\
& \mu_{H}\left(\mathrm{i}_{1}\right)=\{0 / 27.5+0.5 / 33.125+1 / 38.75+0.5 / 44.375+0 / 50\}
\end{aligned}
$$

Where $\mathrm{x}_{\mathrm{i}}$ is the position of the singleton in $\mathrm{i}$ in the universe, and $\mu_{i}$ is equal to the corresponding membership value. This method has a relatively good computational complexity. 
For the rule 12 and 13 , from fig. 3(c), we have $x_{12}=350$ and $x_{23}=475$.

Using Eq. (14), We have

$T C L^{\text {crisp }}=$

$\left[\int_{5}^{16.25}\left(\frac{z-5}{11.25}\right) z d z+\int_{16.25}^{27.5}\left(\frac{27.5-z}{11.25}\right) z d z+\int_{16.25}^{27.5}\left(\frac{z-16.25}{11.25}\right) z d z+\int_{27.5}^{38.75}\left(\frac{38.75-z}{11.25}\right) z d z\right]$

$\div\left[\int_{5}^{16.25}\left(\frac{z-5}{11.25}\right) d z+\int_{16.25}^{27.5}\left(\frac{27.5-z}{11.25}\right) d z+\int_{16.25}^{27.5}\left(\frac{z-16.25}{11.25}\right) d z+\int_{27.5}^{38.75}\left(\frac{38.75-z}{11.25}\right) d z\right]$

$=21.875$

And by using Eq. (15), we have

$$
\text { TCL }^{\text {crisp }}=\frac{16.25 * 19.6875+27.5^{*} 19.6875}{19.6875+19.6875}=21.875
$$

gives the same value as above and from eq.(16), we have

$$
T C L^{\text {crisp }}=\frac{16.25 * 0.5+27.5 * 0.5}{0.5+0.5}=21.875
$$

This just happen to be the same value as above.

The diagram of entire computational fuzzy modeling is presented in fig 7.

SAT $=27.5$

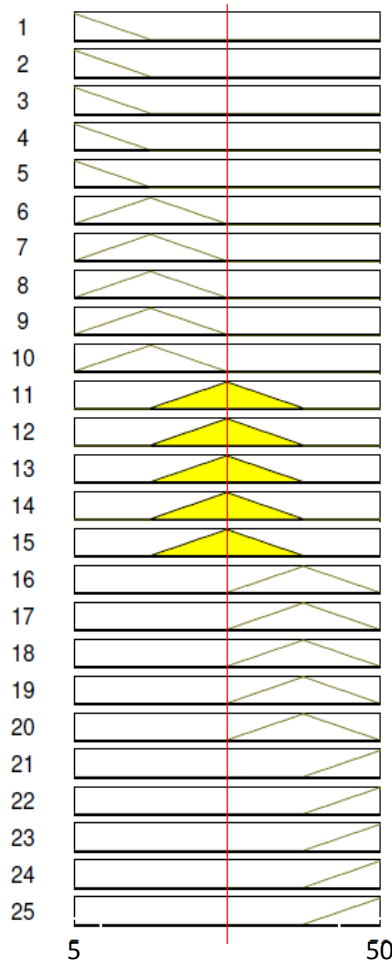

$\mathrm{OTT}=2.19$

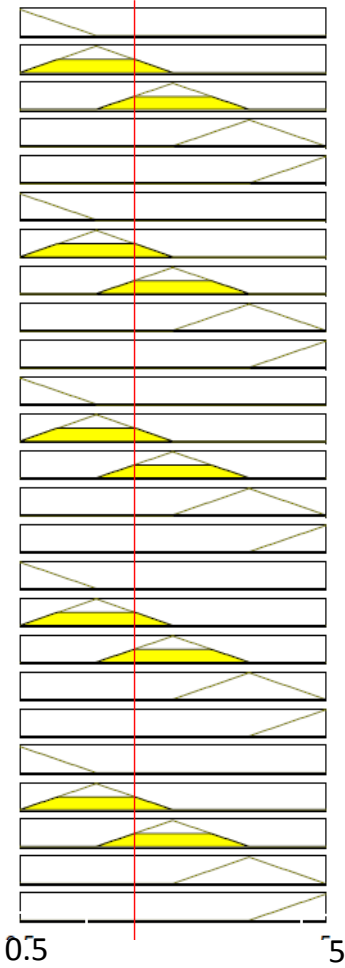

$\mathrm{TCL}=21.9$

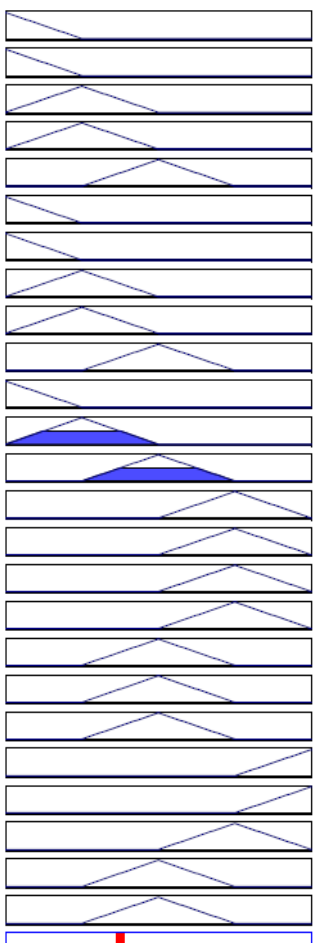

The number of the fuzzy inference engine has a direct upshot on its time intricacy therefore comprising fewer rules may result in improved system performance. Fuzzy rules try to combine these parameters as they are connected in actual world. Some of these rules are mentioned below:

- If SAT is very high and OTT is very high then TCL is Hot

- If SAT is very high and OTT is low then TCL is Comfort

- If SAT is medium and OTT is very low then TCL is Cold

- If SAT is medium and OTT is high then TCL is Slightly Hot.

Once all the variables of OTT and SAT get executed by the FIE, a decision surface is obtained showing the value of the output variable TCL. The decision surface is the dynamic combination of SAT and OTT influencing TCL. Fig 7 presents a three-dimensional curve representing the mapping from SAT and OTT as inputs (axis $\mathrm{X}$ and $\mathrm{Y}$ ) and TCL as an output (axis Z). The temperature, induced inside any of the place due to heat generated caused by the thermal properties of the materials used in building, human metabolism, indoor 
and outdoor surface heat transfer coefficients. In varying situations of SAT and OTT parameters, the maximum value of thermal comfort likely to amend accordingly that depends on the inference engine mechanism.

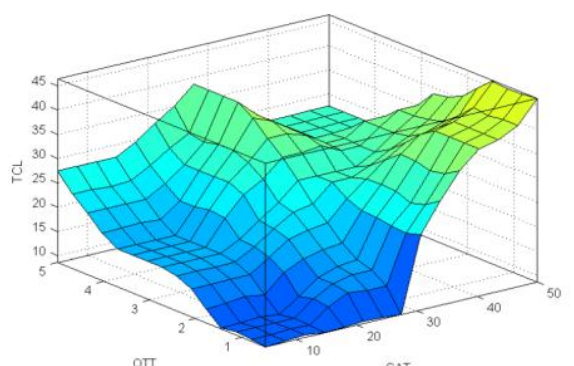

Fig7. Decision surface

According to the fuzzy inference rule, TCL is an output of the corresponding values of SAT and OTT and shown in fig 8 and 9 showing the strong relation between the SAT, OTT and TCL. The nature of the curve is shown when the degree of truth of SAT and OTT is low the degree of truth of TCL remains stagnant and shows that the comfort level is not suitable. As the degree of truth of SAT and OTT increases the degree of truth of TCL raises gradually means that the comfort level is also improving. As OTT and SAT close to 1 TCL remains constant indicates that comfort level is improved and influenced by OTT and SAT.

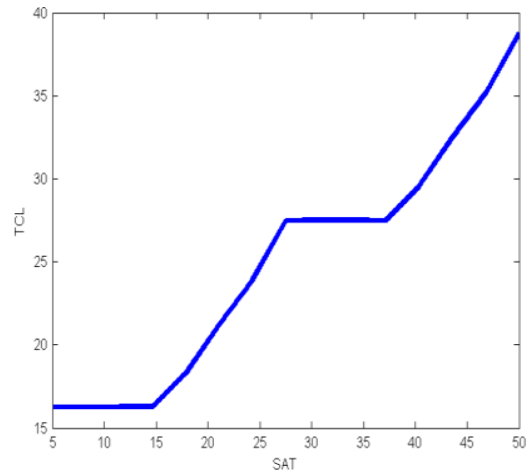

Fig8. Relation between Sat and TCL

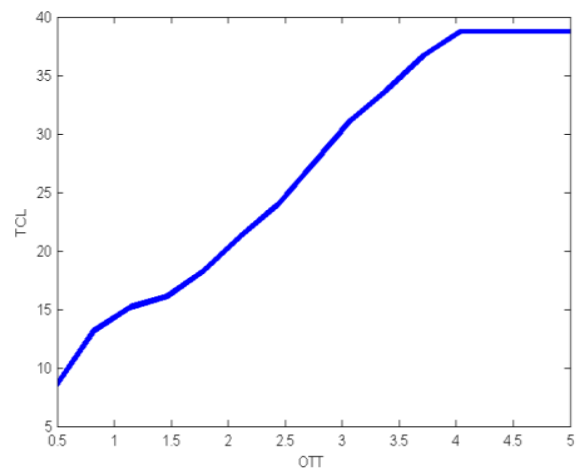

Fig. 9 Relation between OTT and TCL

\section{Conclusion}

In this paper an effort has been made to develop a fuzzy logic approach for the prediction of appropriate TCL considering SAT and OTT as input parameters. In comparison to other predictive modeling techniques, fuzzy models have the advantage of being simple due to the Fuzzy Inference System (FIS) capability to deal with problems that are based on user knowledge and experience. The model will be validated in future through developing the fuzzy rule simulator for real environment.

\section{Acknowledgement:}


It is my immense pleasure and privilege to acknowledge and express my deep sense of gratitude to Dr. P.B.L Chaurasia, Dean, Engineering, Suresh Gyan Vihar University, and Director, Central Building Research Institute, Roorkee who permitted me to carry out this research work successfully.

\section{References:}

[1]. T. Takagi and M. Sugeno, "Fuzzy identification of systems and its application of systems and its applications to modeling and control, "IEEE Trans. Syst., Man, Cybern., vol.15, pp. 116-132,1985.

[2]. E.H Mamdani, "Application of fuzzy algorithms for the control of a dynamic plant, "Proc. IEE, vol.121, pp. 1585-1588, Dec 1974.

[3]. W. Pedrycz and F. Gomide, An introduction to fuzzy sets: analysis and design: The MIT Press, 1998.

[4]. L.A. Zadeh, "fuzzy logic, neural networks, and soft computing, "Commun. ACM, vol.37, pp. 77-84, March 1994.

[5]. L.A Zadeh, "fuzzy sets versus probability, "Proc. IEEE, vol.68, pp.421, March 1980.

[6]. L.A. Zadeh, "fuzzy sets", information and control, vol. 8(3), pp. 338-353, 1965.

[7]. ANSI/ ASHRAE Standard 55, Thermal Environmental Conditions for Human Occupancy.

[8]. Henry Feriadi, and Wong Nyuk Hien, (2003) "Modeling Thermal comfort for Tropics using fuzzy logic", $8^{\text {th }}$ International IBPSA Conference, Netherlands, during Aug 11-14, 2003, pp.323-330.

[9]. Dounis A.I., and Manolakis D.E., (2000) "Design of fuzzy system for living space thermal comfort regulation", Applied Energy, 69(2001), pp.119-144.

[10]. Kosko B. Neural networks and fuzzy systems. Prentice-Hall, 1992

[11]. Lee CC. Fuzzy logic in control systems-fuzzy logic controller. IEEE Trans Systems Man Cybernetics 1990; 20(2):405-35.

[12]. Gupta MM, Kiszka JB, Trojan GM. Multivariable structure of fuzzy control systems. IEEE Trans Systems Man Cybernetics 1986;16(5);638-55.

[13]. Fanger PO. Thermal comfort analysis and applications in environmental engineering. Florida: Publishing Company Malabar, 1982.

[14]. Dounis AI, Santamorious M1, Lefas CC, Argiriou A. Design of a fuzzy set environment comfort system. Energy and Buildings 1995; 22:81-7.

[15]. Dounis AI, Santamorious M1, Lefas CC, Manolakis DE. Thermal-comfort degradation by a visual comfort fuzzy-reasoning machine under natural ventilation. Applied Energy 1994; 48(2):115-30.

[16]. Dounis AI, Santamouris MJ, Lefas CC. Building visual comfort with fuzzy reasoning. Energy Conversion and Management 1993; 34(1):17-28.

[17]. Dounis AI, M1 Santamouris CC. Lefas implementation of A1 techniques in thermal-comfort control for passive solar buildings. Energy Conversion and Management 1992;33(3):175-82.

[18]. ASHARE handbook of fundamentals, physiological principles, comfort and health. Chapter 8, 1985

[19]. D. Misir, H.A. Malki, G.Chen, Design and analysis of a fuzzy proportional-integral-derivative controller, Fuzzy Sets Syst. 79(1996) 297-314.

[20]. Chou Chin-Hsun, Model reference adaptive control: a linguistic space approach, Fuzzy sets system . 96 (1998) 1-20. 